\title{
Infiltrating the Zebrafish Swarm: Design, Implementation and Experimental Tests of a Miniature Robotic Fish Lure for Fish-Robot Interaction Studies
}

\author{
Frank Bonnet $^{1 \dagger}$, Yuta Kato ${ }^{1}$, José Halloy ${ }^{2}$, and Francesco Mondada ${ }^{1}$ \\ ${ }^{1}$ Robotic Systems Laboratory, School of Engineering, \\ Ecole Polytechnique Fédérale de Lausanne, ME B3 30, Station 9, 1015 Lausanne, Switzerland \\ (Tel: +41 2169377 34; E-mail: frank.bonnet@epfl.ch) \\ ${ }^{2}$ Laboratoire Interdisciplinaire des Energies de Demain, \\ Université Paris Diderot VII, Sorbonne Paris Cité, LIED, UMR 8235, F-75205 Paris, France \\ (Tel: +33 (0)1 572753 75; E-mail: jose.halloy@univ-paris-diderot.fr)
}

\begin{abstract}
Robotic fish are nowadays developed for various types of research, such as bio-inspired robotics, biomimetics and animal behavior studies. In the context of our research on the social interactions of the zebrafish Danio Rerio, we developed a miniature robotic fish lure for direct underwater interaction with the living fish. This remotely controlled and waterproof device has a total length of $7.5 \mathrm{~cm}$ with the same size ratio as zebrafish and is able to beat its tail with different frequencies and amplitudes, while following the group of living animals using a mobile robot moving outside water that is coupled with the robotic lure using magnets. The robotic lure is also equipped with an easily rechargeable battery and can be used autonomously underwater for experiments of up to one hour. We performed experiments with the robot moving inside an aquarium with living fish in order to analyze its impact on the zebrafish behavior. We found that the beating rate of the tail increased the attractiveness of the lure among the zebrafish shoal. We also demonstrated that the lure could influence a collective decision of the zebrafish shoal, the swimming direction, when moving with a constant linear speed inside a circular corridor. This new robotic fish design and the experimental results are promising for the field of fish-robot interaction.
\end{abstract}

Keywords: Biomimetics, Bio-Inspired Robotics, Multi-Agent Systems, Animal-Robot interaction

\section{INTRODUCTION}

For humans, social animals can either be considered as a necessity, for instance in the case of a cattle herd, or a threat in the case of a medusa swarm. In both cases, the control of those societies could improve human welfare.

In recent years, with the progress of technology, especially in the robotic field, it became possible to create robotic lures that could generate relevant signals to control an animal society [1]. Once accepted by the society of animals as conspecific, the artificial agents could change the animal collective choice while also adapting to the animal society. One of the first concrete examples was the LEURRE project, where a mixed society consisting of cockroaches and mobile robots was created [2]. These types of experiments have been extended to more complex species, such as beetles [3], crickets [4] or even chicken [5], however, LEURRE was the only study in which the loop of interaction between the robots and the animal collective system was closed.

The group behavior of the zebrafish Danio Rerio, a fish used in many laboratories worldwide for different scientific topics [6], raised the interest of biologists, and several examples of automated lures designed to interact with zebrafish have already appeared. For instance, in [7],[8] and [9], the zebrafish response to a robotic fish with the same ratio size as zebrafish, a beating tail and different colorations was observed. In [10], [11] and [12], a lure attached to a support is moved using a mobile robot outside the aquarium and controlled using a tracking soft-

$\dagger$ Frank Bonnet is the presenter of this paper. ware. To our knowledge, none of these studies showed a significant acceptance of the artificial agents by the living fish.

In this study, we propose an innovative system to interact with the shoal of fish. We used biomimetics approach to design a robotic fish lure for direct underwater interaction with fish: RiBot, which is only 1.8 times bigger than a standard size zebrafish, with the same ratio dimensions (RiBot is a combination of the word Riba that means fish in Russian language and the word Robot). It is composed of an actuated tail (caudal peduncle) that can beat with frequencies up to $20 \mathrm{~Hz}$ and amplitude of $+/$ 23 degrees, which are in the range of the caudal peduncle beating movements measured on our own zebrafish. RiBot is waterproof and totally autonomous with an internal Lithium Polymer (LiPo) battery and Infrared (IR) receptor for wireless control. Furthermore, RiBot can be magnetically coupled with an external mobile robot, FishBot, developed during preliminary research and that can reach similar speeds and accelerations to zebrafish ([13] and [14]). When compared to previously published solutions, our solution is combining a mobile robot outside of water as presented in [10]-[12] and a robotic lure as in [7]-[9] with smaller size. This allows us to have many different stimuli generated by the device to monitor the zebrafish society.

We performed experiments using this robotic system and measured its impact on the fish behavior. We measured the fish attractiveness while varying the amplitude and the frequency of the beating tail of RiBot, while moving it inside the aquarium at the same average speed as 
zebrafish. Results from these experiments will help scientists to better understand the types of interaction between fish that lead to formation of shoal and to collective choices.

\section{HARDWARE DESIGN}

The design of underwater autonomous vehicle is always of great challenge, especially at very low scale. The device should be waterproof, wireless and, moreover in the case of this study, the robot has to interact with zebrafish and thus its size shall be in the range of zebrafish, whose average length rarely exceed $45 \mathrm{~mm}$ in laboratory conditions. As shown in [7] zebrafish can be attracted by a bigger replica fish as soon as the latter has the same ratio size as zebrafish. Due to the size of the selected components, the length of RiBot was fixed at $75 \mathrm{~mm}$ while keeping the same ratio size as zebrafish, with a width of $10 \mathrm{~mm}$ and a height of $17 \mathrm{~mm}$. For the first prototype, we have decided to include only one actuator, a stepper motor to actuate the tail, a rechargeable battery LiPo to allow the energy autonomy of the device and an infrared receiver to remotely control the device underwater. Figure 1 shows the different hardware subsystems of RiBot that will be described in the following subsections.

\subsection{Actuator}

There are several solutions that can be found in the literature to actuate a fish-like underwater vehicle tail or fin [15]. Concerning RiBot, a very small actuator that consumes very low energy but with enough torque to actuate a robotic fish caudal peduncle underwater was required. The actuator should also allow beating tail frequencies and amplitudes in the range of the zebrafish.

We considered a kinematic fish model shown in Fig. 2 to determine the required motor specifications. To simplify the model, we assumed that the caudal peduncle is a rigid body that does circular motion centered on the motor shaft.

If $\theta$ is the angle between the tail and the longitudinal axis, the motion equation can be expressed by Eq. 1,

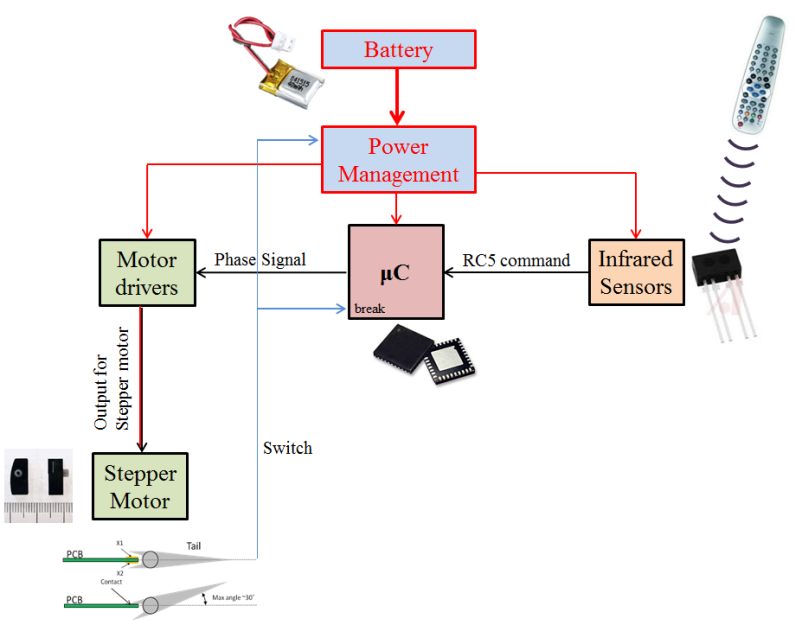

Fig. 1 Hardware schematic of RiBot. where $I$ is the inertial moment of the caudal peduncle, $L$ is the length of the caudal peduncle, $F_{d}$ is the driven force of the motor, $F_{w}$ is the resistance of the water that is approximated as the drag force only (Eq. 2) and $F_{e}$ is the resistance force of the elastic skin with coefficient $k$ that envelop the caudal peduncle and this resistance will thus be unbalanced on each sides of the caudal peduncle (Eq. 3).

$$
\begin{aligned}
I \ddot{\theta} & =\frac{F_{d} L}{2}-\frac{F_{w} L}{2}-\frac{F_{e} L}{2} \\
F_{w} & =\frac{1}{2} C_{D} A \rho\left(\frac{L \dot{\theta}}{2}\right)^{2} \\
F_{e} & =\frac{k L \theta}{2}
\end{aligned}
$$

Using Eq. 1-3, we obtained a minimal value of $2 \mathrm{mNm}$ for the torque needed for the actuator and selected a micro step gear motor "MF03G" of Seiko Precision Inc. to fulfill this. The specifications of this 2 phases stepper motor are shown in Tab. 1. The motor has very small dimensions (Fig. 3) and can thus be easily integrated inside RiBot design. The advantage of using a stepper motor is that if the motor does not miss any steps, the position of the caudal peduncle can be estimated from the number of pulses emitted to drive the motor.

\subsection{Communication}

IR communication was chosen to remotely control RiBot. Indeed, Wi-Fi and bluetooth involve bulky chips and more power resources which was not affordable for this design. An IR sensor is placed on the back of RiBot (Fig. 6) and IR signal can be sent from any direction to control the device underwater. The RC5 protocol is implemented and thus universal TV remote control with

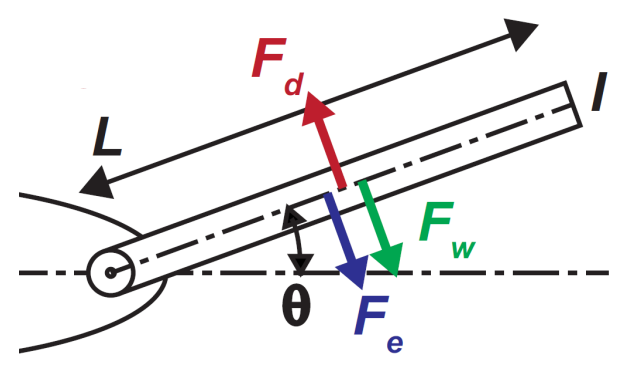

Fig. 2 The model of the tail (caudal peduncle) used to estimate the needed torque for the actuator.

Table 1 Micro step gear motor "MF03G" characteristics.

\begin{tabular}{|c|c|}
\hline Characteristics & Value \\
\hline Motor type & 2 phase stepper motor \\
\hline Size & $13.3 \times 6.5 \times 7.4 \mathrm{~mm}$ \\
\hline Mass & $0.6 \mathrm{~g}$ \\
\hline Min. step angle & $0.172 \mathrm{deg}$ \\
\hline Voltage & $3 \mathrm{~V}$ \\
\hline Max. speed & $1200 \mathrm{step} / \mathrm{s}$ \\
\hline Internal resistance & $28.5 \Omega$ \\
\hline Torque & Min. $2 \mathrm{mNm}$ \\
\hline
\end{tabular}


RC5 protocol can be used to control the device. RiBot was placed below a $10 \mathrm{~cm}$ water layer and the signal, emitted at $2 \mathrm{~m}$ from the water surface, could be perceived by the robot.

\subsection{Power}

In Sec. 2.1 we described the stepper motor selected to drive the caudal peduncle with the required voltage and current. As the other parts of the electronic had very low power consumption, the motor power consumption was used to select the capacity of the battery $(C)$ using the following equation

$$
C=\frac{V}{R} T
$$

where $V$ is the necessary voltage, $R$ is the internal resistance of the motor and $T$ is the desired duration of the experiment which was set to 30 minutes.

We obtained a required value of $52 \mathrm{mAh}$ in capacity. As it was not possible to find a battery with very small size and the required capacity, we selected the SparkFun small LiPo battery which characteristics are described in Tab. 2. This battery is rechargeable, which gives the advantage that we do not need to change it on the device when it is empty. Indeed, the battery can be recharged through the eyes of the fish that are made of brass and that cross the Polyurethane layer to be accessed from the outside of RiBot (Fig. 6). A charge circuit (Fig. 4) was designed in order to manage the powering of the device and the recharging of the battery.

\subsection{Electronic design}

The main skeleton of RiBot consists of a Printed Circuit Board (PCB) of $1.6 \mathrm{~mm}$ width. All the components are soldered on the two faces of the PCB and are encapsulated with an impermeable coating made of Polyurethane.

The tail can be used for two purposes (Fig. 5): It can turn $\mathrm{ON}$ and OFF the device and also can be used to

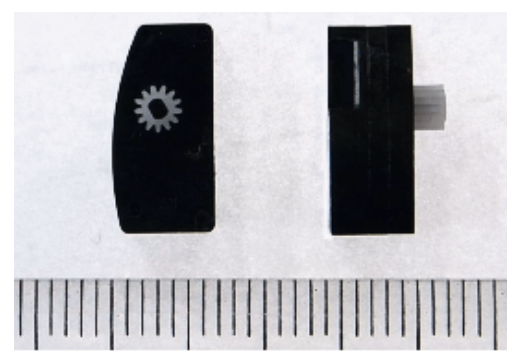

Fig. 3 Stepper motor MF03G of Seiko Precision Inc. selected to actuate the caudal peduncle of RiBot.

Table 2 Sparkfun LiPo battery characteristics.

\begin{tabular}{|c|c|}
\hline Characteristics & Value \\
\hline Battery type & polymer lithium ion \\
\hline Size & $12 \times 16 \times 5 \mathrm{~mm}$ \\
\hline Mass & $2 \mathrm{~g}$ \\
hline Capacity & $40 \mathrm{mAh}$ \\
\hline Voltage & $3.7 \mathrm{~V}$ \\
\hline
\end{tabular}

calibrate the tail position, thanks to the circuit shown in Fig. 4. When the tail reaches one of its maximal position (Fig. 5.b and Fig. 5.c), a contact is made between the pad $X 1$ or $X 2$ and the tail $X 3$, which is made of conductive material (brass) and connected to the ground $(G N D)$ as shown in Fig. 4. When RiBot is turned OFF and the tail reaches one of the two pads, the /EndCourse section is at the GND, thus the /PowerOn is also at the GND and the transistor T1 conducts. $V_{S y s}$, which power supply the system which is then at the battery voltage $V_{B a t}$ minus the junction voltage of the transistor $V_{S D}$. Then, the microcontroller is switched on and at the beginning of

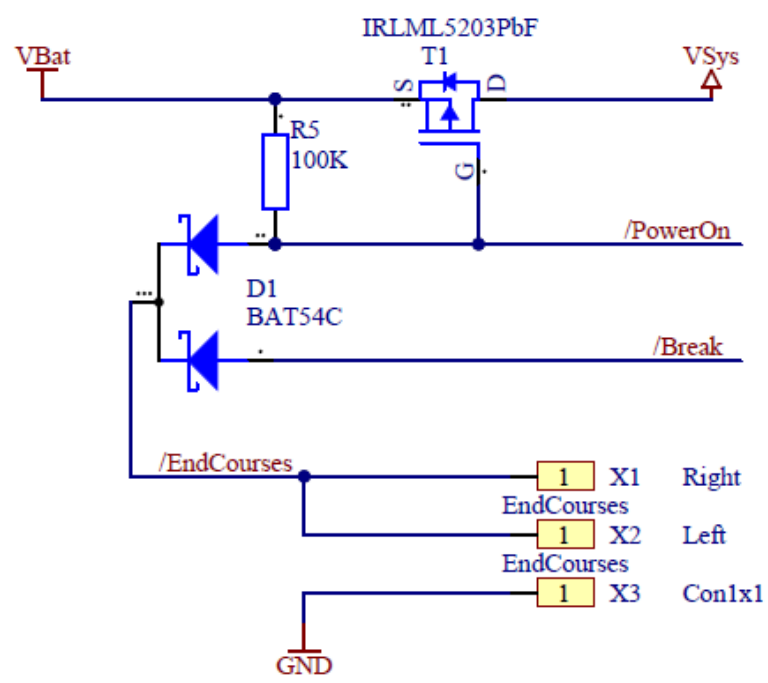

Fig. 4 Electronic schematic of the power management system. A dual-diode system is used to change the state of the transistor $\mathrm{T} 1$ to switch the robot $\mathrm{ON}$. The system is also used to calibrate the tail position (Fig. 5).

(a)

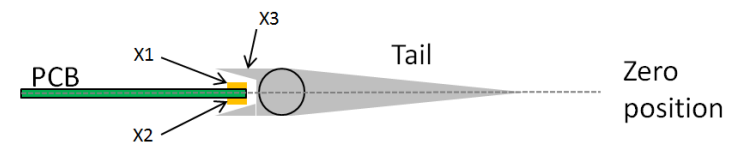

(b)

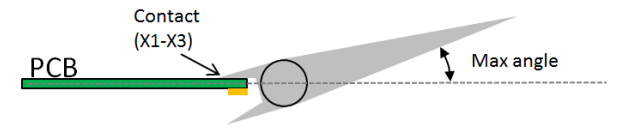

(c)

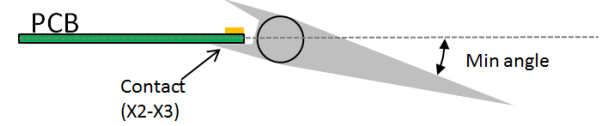

Fig. 5 Schematic of the switch system for the tail. The actuator is fixed on the PCB and actuates the tail. Two contact pads ( $X 1$ and $X 2$ ) on the PCB will allow a contact with the fin when it reaches one of the two maximal position $(b, c)$. This will either turn $\mathrm{ON}$ the device if it was turned OFF or either generate a break on the microcontroller in order to calibrate the fin position to find the zero position (a). 
the code, the / PowerOn connected to an output of the microcontroller is forced to 0 which maintains the device $\mathrm{ON}$ even if a new contact is made between the tail and one of the pads. From this point, the tail is used for the break function implemented in the microcontroller (/Break) in order to calibrate the tail initial position. Indeed, there are no sensors placed in order to retrieve the position of the stepper motor, and due to the fact that the stepper motor might lose steps, one has to recalibrate sometimes the tail position, and the contact made between pads $X 1$ or $X 2$ and $X 3$ is used to determine the two maximal position of the tail, and thus the zero position located in the middle of these two extreme positions.

The PCB carries a microcontroller STM32f103. This microcontroller was selected due to its very small dimensions ( $6 \mathrm{~mm} \times 6 \mathrm{~mm}$ ) and its functionalities. It is an ARM Cortex-M3, with 128KB Flash memory and 16KB Random Access Memory (RAM) and enough General Purpose Inputs and Outputs (GPIOs).

The actuator is driven using a dual full-bridge A3901 also selected for its small dimensions ( $3 \mathrm{~mm}$ x $3 \mathrm{~mm}$ x $0.75 \mathrm{~mm})$, its operating range for voltage $(2.5 \mathrm{~V}-5.5 \mathrm{~V})$ and current $( \pm 400 \mathrm{~mA})$.

An IR sensor TSOP75436WTT is installed on the top of RiBot, to retrieve IR signal from a wireless remote control.

During assembly, all the components were soldered in an oven except for the IR receiver and for the eyes. The IR receiver is placed on the edge of the PCB in order that its sensitive side faces the top of the fish lure to better capture the IR signals coming above the aquarium. A programming connector is soldered on one side of RiBot and is used for flashing the firmware and debug purposes.

\subsection{Mechanical design}

As mentioned in the previous subsection, the skeleton of RiBot consists of a PCB on which all the main mechanical and electronic components are attached. The motor with the tail docked on its axis is fixed inside a 3D part, called the ring as it has an elliptical external shape, that is glued on the PCB.

In order to isolate the actuator and the caudal peduncle from water and to create a soft skin that can mimic the tail of the zebrafish, an undercut of the tail was made in $3 \mathrm{D}$ printing and was dipped into liquid latex. The skin created was unmolded and attached on the ring using silicone. The caudal fin is also made of latex and is prepared apart from the tail using another mold, and is glued on the tail using latex.

Polyurethane is used to isolate the electronics from water. A mold with the desired undercut of Ribot was made of ABS by 3D printing (Fig. 7). The mold is composed of two parts that are joined using pins and screws during the molding process to press the two parts against each other. The PCB is placed inside the mold and the eyes are used as a reference inside the mold. Finally. liquid polyurethane is injected inside the mold from the tail. The Polyurethane coats the entire PCB up to the ring and hardens inside the mold. After this process, RiBot is to- tally isolated from water and can start to swim underwater (Fig 8).

\section{PERFORMANCE EVALUATION}

Figure 8 shows the prototype of RiBot in comparison with one of our zebrafish DanioRerio. The final prototype has a mass of 9.0 grams and sinks beneath the water

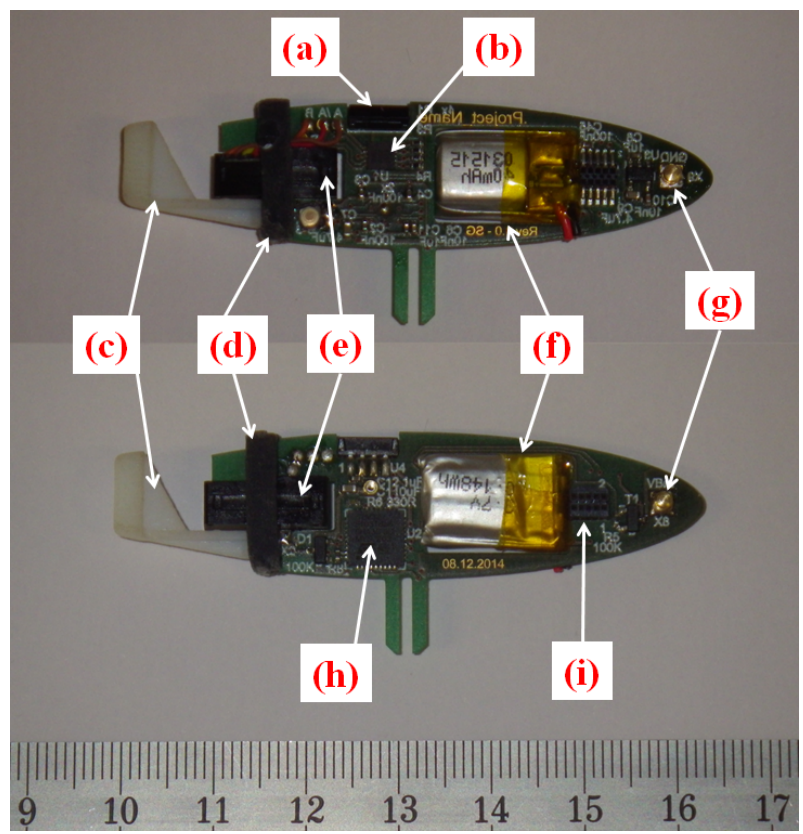

Fig. 6 Skeleton of RiBot: a) IR receiver. b) Dual Hbridge A3901. c) Tail (caudal peduncle). d) Ring. e) Stepper motor. f) LiPo battery. g) Eyes used as contacts to recharge the battery and reference during molding. h) Microcontroller STM32f103. i) Connector used for debug purposes.

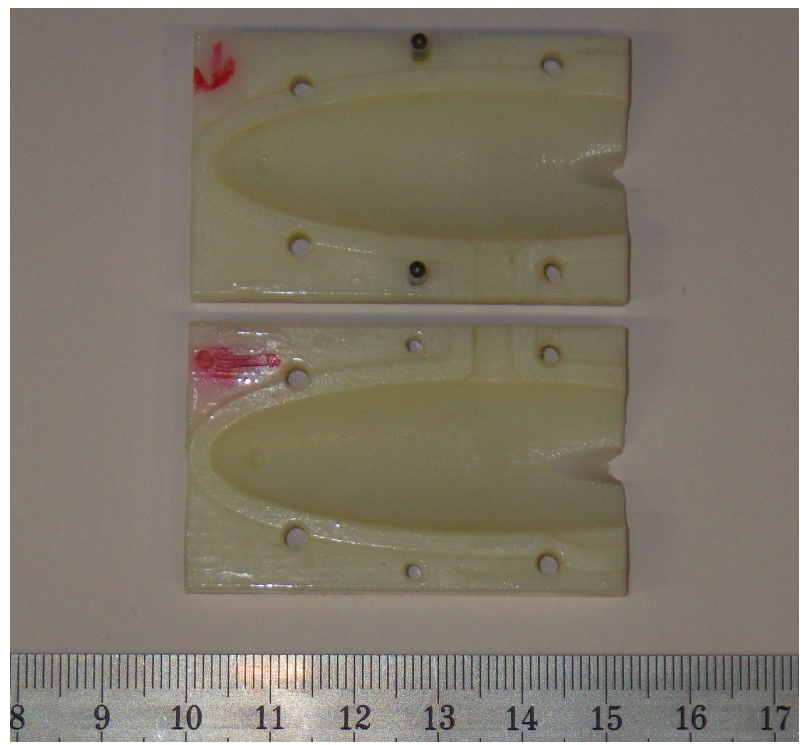

Fig. 7 Mold used for molding RiBot with Polyurethane. The two parts are aligned with pins and then screws are used to press the two parts of the mold against each others. 
surface due to its density.

\subsection{System qualifications}

The first prototype of RiBot presented in Fig. 8 was inserted inside water in order to measure its capabilities.

We could perform 13 minutes long test with the tail of RiBot moving continuously using the embedded LiPo battery. This is a little bit under the expected time of the experiment that was presented in the specifications of the battery (Sec. 2.3) and this is due to the fact that the battery has a smaller capacity than the one required and the motor consumes slightly more current than what is indicated in its specifications. However, when reducing the use of the actuator, RiBot could be maintained turned $\mathrm{ON}$ for more than one hour underwater.

In order to measure the beating tail of RiBot, we used the same experimental setup that was used to perform the experiments with fish presented in Fig. 10. We installed a color marker on the edge of the tail and above the position of the actuator axis and tracked these markers from top view using a camera. We could thus measure the position of the edge of the tail relative to the position of the motor axis, and we could verify the amplitudes and frequencies obtained.

We obtained a maximum amplitude of 23 degrees and a frequency of $20 \mathrm{~Hz}$. This corresponds to the amplitude and vibrations that we have observed on our own zebrafish. Indeed, in rare cases, the zebrafish can move the tail at an angle of almost 90 degrees, but in general, the amplitude does not exceed 20 degrees.

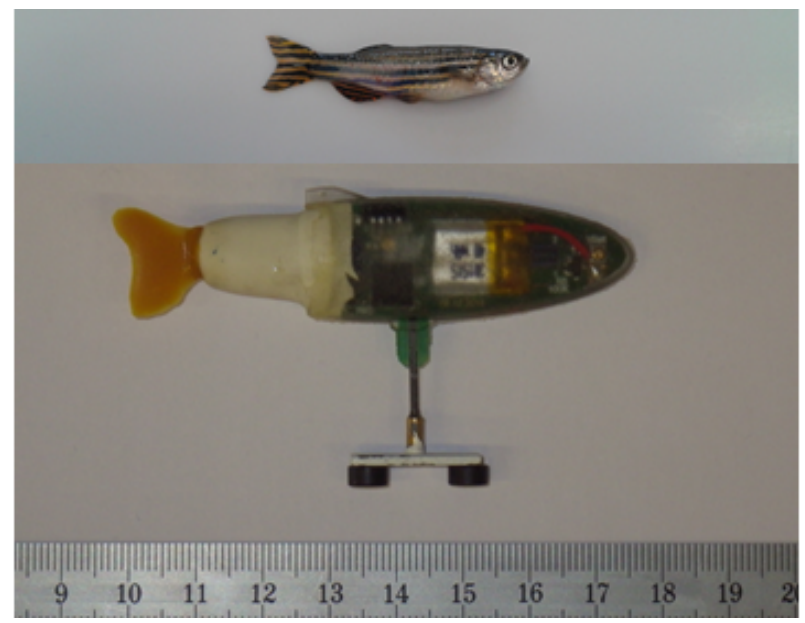

Fig. 8 The prototype of RiBot, compared with a zebrafish Danio Rerio used during the experiments. This figure shows the 1.8 ratio between the lure and a real zebrafish. RiBot is equipped with the Latex socket enclosing the tail and was molded inside Polyurethane to isolate the PCB from water. RiBot is mounted on a base composed of a tiny carbon stick and an iron plate. Magnets are attached to the iron plate to magnetically couple RiBot with a mobile robot moving below the experimental tank.

\subsection{Motion underwater}

RiBot was primarily designed to simply beat its tail in order to increase its attractiveness towards real zebrafish. However, we discovered that the actuated tail could also be used to propel RiBot underwater autonomously without using another robot to propel it. The one degree of freedom actuator, which actuates the caudal peduncle, coupled with the thin caudal fin (Fig 8), allow the device to move autonomously underwater with speeds up to $2.5 \mathrm{~cm} / \mathrm{s}$, using a floating element to stabilize it as no elements to control buoyancy are implemented yet. This maximal speed is in the range of some results obtained with micro underwater vehicles [16][17].

The maximal angular speed of the tail $\omega_{\max }$ was obtained using the maximal angular speed of the stepper motor $V_{\max }=1200 \mathrm{steps} / \mathrm{s}$. Microstep mode was employed thus one step corresponds to $\alpha_{\text {step }}=$ $0.171 \mathrm{deg} / \mathrm{step}$ and one could obtain de maximal angular speed

$\omega_{\max }=V_{\max } * \alpha_{\text {step }}=205.2 \mathrm{deg} / \mathrm{s}$

The maximal beating rate could be determined from the desired amplitude of tail beating $A_{d e s}$. The maximal beating rate $f_{\text {beat,max }}$ is given by the maximal angular speed divided by 4 times the desired amplitude to obtain one beating cycle.

$f_{\text {beat }, \max }=\frac{\omega_{\max }}{4 * A_{\text {des }}}$

Using this formula, we measured the linear speed of RiBot underwater with all the different possible amplitudes and frequencies. One can observe in Fig 9 that RiBot swims slightly faster at high amplitude than at high frequency. The maximal speed of $25 \mathrm{~mm} / \mathrm{s}$ is obtained at a amplitude of $22 \mathrm{deg}$ and a beating rate of $1 \mathrm{~Hz}$.

\section{BEHAVIORAL EXPERIMENTS}

In order to qualify RiBot for behavior studies with zebrafish, we designed an experiment to test the attractiveness of the device among a group of living zebrafish.

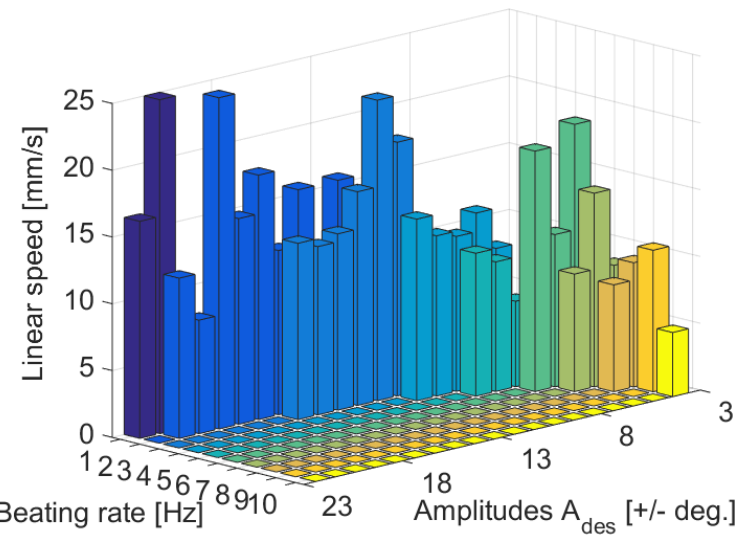

Fig. 9 Average linear speed of RiBot underwater in function of the amplitude and the beating rate of the tail. The value of 0 corresponds to the cases beyond the motor capabilities and thus impossible. 


\subsection{Experimental setup}

The experimental setup that is used for the experiments consists of an aquarium of $1000 \mathrm{~mm} \times 1000 \mathrm{~mm}$ of surface covered on the inside with white teflon sheets (Fig. 10). These sheets are installed in order to avoid reflection on the glass and to have a smooth surface for the motion of the lure module inside the aquarium. The tank is filled with water up to a level of $60 \mathrm{~mm}$. According to [6], this level of water is not introducing more stress for the fish and furthermore, the lure, whose height cannot vary, will be more visible for the fish that are swimming around. The water temperature is set to $26^{\circ} \mathrm{C}$, as suggested by [6]. A mobile robot, FishBot, designed also in our laboratory, is moving under the aquarium, and the motion is transmitted to RiBot using magnets. FishBot is powered using two conductive plates, one glued on the bottom of the aquarium and one on the support on which the mobile robot is moving.

Inside of the tank, a structure made of white teflon bands of $1 \mathrm{~mm}$ width and $8 \mathrm{~cm}$ height constrains the fish into a $10 \mathrm{~cm}$ large circular corridor (Fig. 11).

\subsection{Tracking}

During the experiments, video frames are grabbed by a high-definition webcam placed on top of the setup. The frames are retrieved on a computer and a blob detection based algorithm retrieves the position of RiBot and the zebrafish (Fig. 11). The data acquired during the experiments are then processed using Matlab.

\subsection{Animals}

The experiments performed in this study were conducted under the authorization $\mathrm{N}^{\circ} 2778$ delivered by the Department of Consumer and Veterinary of the Canton

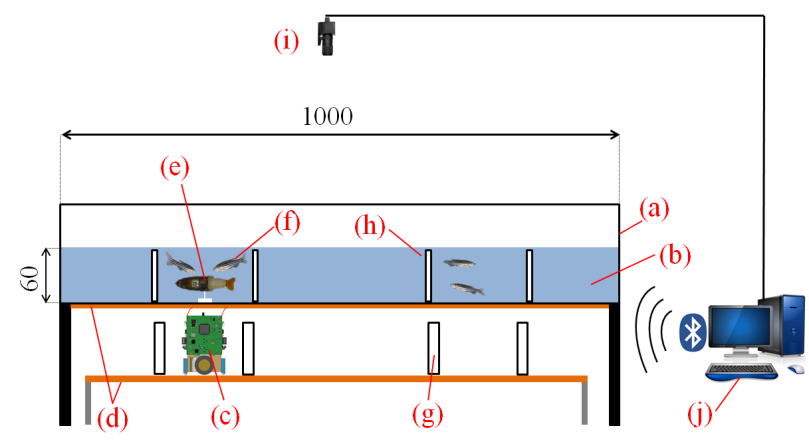

Fig. 10 Experimental setup used during the experiments. a) Aquarium of $1000 \mathrm{~mm} \times 1000 \mathrm{~mm} \times 250 \mathrm{~mm}$. b) Water layer of $60 \mathrm{~mm}$ depth. c) FishBot mobile robot moving under the aquarium. d) Conductive plates to power the mobile robot. e) RiBot inside the aquarium linked to the mobile robot through magnetic coupling. f) Living zebrafish. g) Walls of the circular corridor to constrain the mobile robot. h) Walls of the circular corridor to constrain the zebrafish i) Camera used to track the lure and the zebrafish. j) Computer that process the camera frames and remotely controlled the robot via Bluetooth protocol. de Vaud (Switzerland) after submission to the state ethical board for animal experiments.

For the experiments performed, we used 50 wild-type zebrafish Danio Rerio, with short fins. These zebrafish were acquired in a pet shop, and are stored in a 60 litres housing aquarium. The average total length of our zebrafish was $\sim 40 \mathrm{~mm}$. The water temperature of the housing aquarium is $26^{\circ} \mathrm{C}$. The fish were fed twice a day using a food distributor with commercial food. The enrichment in the aquarium consisted of plastic plants, cladophoras, gravel, rocks and aquatic snails.

\subsection{Experiment design}

We tested 5 different modes for the beating tail of RiBot (Tab. 3) and three different linear constant speeds of the mobile robot: $0,4 \mathrm{~cm} / \mathrm{s}$ and $8 \mathrm{~cm} / \mathrm{s}$. We have tested all different conditions with 8 trials randomlyinstantiated of one minute each. We used 5 zebrafish inside the circular corridor during the experiment that were picked randomly on the housing aquarium. The fish could thus move either clockwise $(\mathrm{CW})$ or counter-clockwise $(\mathrm{CCW})$ and RiBot was moving only $\mathrm{CCW}$ in order to determine if it could influence the fish to move inside the corridor with the same direction. After every 10 minutes of experiments, the group of five fish was replaced by another group of five zebrafish. We did not considered the visual aspect of RiBot in our experiment even if it is well known that it is an important factor in the attraction of zebrafish [7]. This parameter will be tested in future experiments.

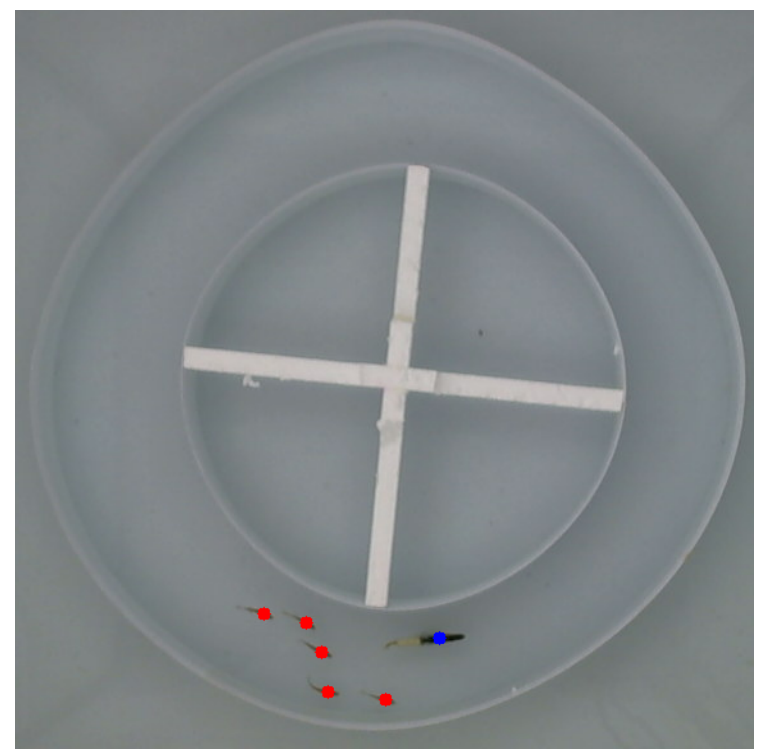

Fig. 11 The experimental setup grabbed from the top by the high-definition webcam, with the result of the blob detection based software. RiBot (in blue) can be easily differentiated from the five zebrafish (in red) thanks to its larger size. The inner circle and the outer circle have a diameter of approximatively $40 \mathrm{~cm}$ and $60 \mathrm{~cm}$ respectively, thus the fish are constrained in a corridor of $10 \mathrm{~cm}$ in average. 


\section{RESULTS AND DISCUSSION}

In our experiments, we have considered two types of measurements to characterise the acceptance of RiBot: The percentage of time that zebrafish are located at a distance below $5 \mathrm{~cm}$ from RiBot, and the swimming direction of the zebrafish inside the ring shape setup, which is either CW or CCW. The first measurement is a good indication of the acceptance of a robotic device by the group of fish [7][13], compared to the distance between the fish and the robot position, as for the latter we usually obtained less significant effect as we shown in [13]. The second measurement offers a good indication of the influence of RiBot on the collective decision of the group of zebrafish.

Figure 12 shows the percentage of time spent by zebrafish in a $5 \mathrm{~cm}$ radius distance from $\mathrm{RiBot}$ when the latter was beating its tail, for the three different linear speeds of the robot. When the tail is not beating (frequency of $0 \mathrm{~Hz}$ ), the presence of zebrafish near RiBot does not depend on the robot linear speed. The standard deviation in these conditions is also relatively small. However, when increasing the frequency of vibration of the tail, we can observe that the impact on the fish attraction started to depend on the robot linear speed. Indeed, when RiBot is static, its attractiveness increase significantly with the increase of vibration, compare to the case when RiBot is moving for which the increase of attraction is not significant. It could be postulated that when RiBot is moving, it also generates vibration underwater, and thus the change of vibration perceived by the zebrafish is not as significant as when RiBot is static. But for the latter, we can argue that the increase of tail beating frequency has a small impact on the attraction of the fish. The increase of attractiveness of an actuated robotic lure towards a group of fish was already mentioned in [9], in which it was demonstrated that fish were more attracted towards the robot when its tail was beating rather than when it was not. It can also be noticed that the standard deviation is increasing with the increase of beating frequency. This could be explained by the fact that this factor has an effect on certain group of fish, but this effect is not constant. Another explanation of this effect can be that the zebrafish may get bored after a certain period of time with the tail beating of RiBot and thus after a short period of attraction, their interest decreases.

Table 4 shows the swimming direction of the zebrafish for every linear speed conditions of RiBot. The direction was determined using the position of the fish retrieved

Table 3 The five different modes for the beating tail that were used during the experiments.

\begin{tabular}{|l|c|c|}
\hline Mode ID & Vibration [Hz] & Amplitude [+/- deg.] \\
\hline 1 & 0 & 0 \\
2 & 1 & 20 \\
3 & 2 & 15 \\
4 & 5 & 5 \\
5 & 10 & 2 \\
\hline
\end{tabular}

by the tracking software. The corridor was divided into four quadrants and when a fish passes from one quadrant to the other, depending on the direction, it is counted as swimming $\mathrm{CW}$ or CCW. Without any device present inside water, we noticed that the group of zebrafish swims half of the time in one direction $(\mathrm{CW})$ and half of the time in the other direction (CCW) so the setup has no bias. The experimental result shown in Tab. 4 indicates that RiBot can influence the collective decision of a group of five zebrafish when moving inside a circular corridor. Indeed, when RiBot is not moving (linear speed $=0 \mathrm{~cm} / \mathrm{s}$ ), the zebrafish swims half of the time $\mathrm{CW}$ and half of the time $\mathrm{CCW}$ as for the case when no robotic devices are present. When RiBot is moving with a linear speed of $4 \mathrm{~cm} / \mathrm{s}$ with a CCW direction, the zebrafish start to swim more in the $\mathrm{CCW}$ direction, and this effect increase when RiBot is moving with a linear speed of $8 \mathrm{~cm} / \mathrm{s}$ with the zebrafish swimming $\mathrm{CCW} 70 \%$ of the time with very low standard deviation. This result demonstrates that the robotic system composed of FishBot and RiBot is able to change the collective decision of the zebrafish shoal, i.e. the swimming direction. Further works will investigate more deeply the factors that could generate this effect, but this is a promising result using a prototype version of $\mathrm{RiBot}$.

\section{CONCLUSION AND FUTURE WORKS}

This paper introduced the design of a new robotic fish lure, RiBot, for fish-robot interaction studies. This device could also be used for different research areas such as

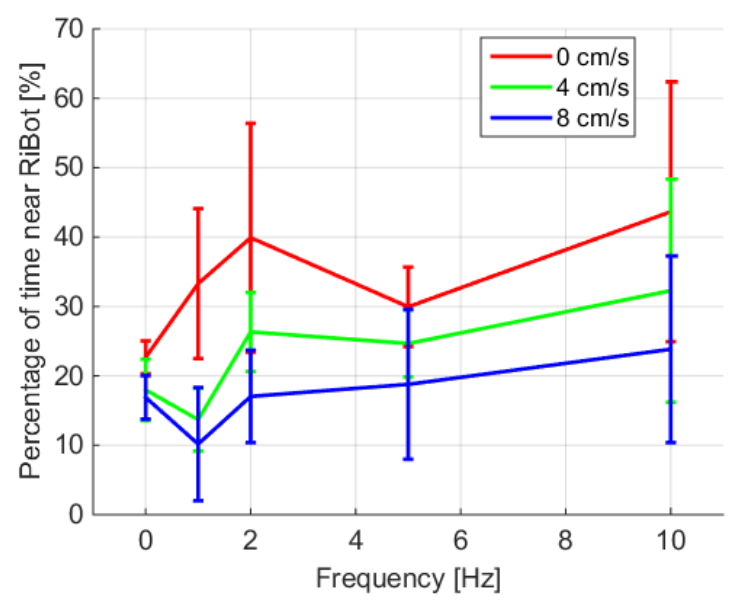

Fig. 12 Presence of zebrafish at a distance of $5 \mathrm{~cm}$ from the robotic system for the three different linear speeds. This was measured using the tracking software that retrieved for each video frame the position of RiBot and the zebrafish.

Table 4 Direction in which the zebrafish are swimming $((\mathrm{CW})$ or $(\mathrm{CCW}))$, with RiBot not placed in the experimental setup or present and moving only $\mathrm{CCW}$.

\begin{tabular}{|l|c|c|c|}
\hline Robot lin. sp. [cm/s] & CCW [\%] & CW [\%] & Std. dev. [\%] \\
\hline Without RiBot & 52.1 & 47.9 & 5.4 \\
0 & 50.8 & 49.2 & 7.1 \\
4 & 60.0 & 40.0 & 8.6 \\
8 & 70.7 & 29.3 & 6.5 \\
\hline
\end{tabular}


bio-inspired robotics, biomimetics and animal behavior studies.

We showed that the first prototype of RiBot is able to beat its tail with amplitude up to 23 degrees and frequency up to $20 \mathrm{~Hz}$. The device has an autonomy of 13 min when the actuator is continuously active during the experiment, but can reach more than one hour if the actuated tail is only intermittently used. We also measured the speed of the lure when varying the tail beating frequency and amplitude and obtained a maximum linear speed of RiBot underwater of $2.5 \mathrm{~cm} / \mathrm{s}$.

Behavioural experiments, conducted using RiBot coupled with a mobile robot in order to reach zebrafish typical linear speed, indicated that the speed of RiBot can influence the collective choice of zebrafish. This is a promising result as no other studies to our knowledge has shown such results in the case of behavioral studies on zebrafish Danio Rerio.

Several improvements will be made in future work to decrease the size of RiBot, and several electronic components, such as LEDs and speakers will be added to generate more stimuli to increase the attractiveness of the device among zebrafish. Experiments involving a swarm of robotic agents will also be performed in order to study the interaction between a group of robots and a group of fish.

\section{ACKNOWLEDGEMENTS}

This work was supported by the EU-ICT project ASSISIbf, No. 601074. The information provided is the sole responsibility of the authors and does not reflect the European Commissions opinion. The European Commission is not responsible for any use that might be made of data appearing in this publication. We also thank Daniel Burnier and Norbert Crot (LSRO) for the technical support during RiBot design and production.

\section{REFERENCES}

[1] F. Mondada, A. Martinoli, N. Correll, A. Gribovskiy, J. I. Halloy, R. Siegwart, and J.-L. Deneubourg. A general methodology for the control of mixed natural-artificial societies. In S. Kernbach, editor, Handbook of Collective Robotics, Chapter 15, pages 547-586. Pan Stanford Publishing, Singapore, 2013.

[2] J. Halloy, G. Sempo, G. Caprari, C. Rivault, M. Asadpour, F. Tache, I. Said, V. Durier, S. Canonge, J. Ame, C. Detrain, N. Correll,A. Martinoli, F. Mondada, R. Siegwart, and J.-L. Deneubourg, Social Integration of Robots into Groups of Cockroaches to Control Self-Organized Choices, Science, vol. 318, no. 5853, pp. 1155-1158, 2007.

[3] J. Krause, A. Winfield, and J.-L. Deneubourg, Interactive robots in experimental biology, Trends in Ecology and Evolution, vol. 26, no. 7, 2011.

[4] K. Kawabata, H. Aonuma, Ko. Hosoda, and J. Xue, A System for Automated Interaction with the Cricket Utilizing a Micro Mobile Robot, Journal of Robotics and Mechatronics Vol.25 No.2, 2013
[5] A. Gribovksiy, J. Halloy, J.-L. Deneubourg, and F. Mondada, The poulbot, a mobile robot for ethological studies on domestic chickens, Symposium on AIInspired biology, 2010.

[6] B. Reed and M. Jennings, Guidance on the housing and care of Zebrafish Danio Rerio. Research Animals Department, Science Group, RSPCA, 2010.

[7] N. Abaid, T. Bartolini, S. Macri, and M. Porfiri, Zebrafish responds differentially to a robotic fish of varying aspect ratio, tail beat frequency, noise and color, Behavioural Brain Research, vol. 233, pp. 545553, 2012.

[8] M. Aureli, F. Fiorilli, and M. Porfiri, Portraits of selforganization in fish schools interacting with robots, Physica D: Nonlinear Phenomena, vol. 241, no. 9, pp. 908-920, 2012.

[9] S. Marras and M. Porfiri, Fish and robots swimming together: attraction towards the robot demands biomimetic locomotion, J. R. Soc. Interface, Aug 7;9(73):1856-68, 2012.

[10] J. Faria, J. Dyer, R. Clement, I. Couzin, N. Holt, A. Ward, D. Waters, and J. Krause, A novel method for investigating the collective behaviour of fish: Introducing Robofish, Behavioral Ecology and Sociobiology, vol. 64, pp. 1211-1218, 2010.

[11] D. T. Swain, I. D. Couzin, and N. E. Leonard, Realtime feedbackcontrolled robotic fish for behavioral experiments with fish schools. Proceedings of the IEEE, vol. 100, no. 1, pp. 150163, 2012.

[12] T. Landgraf, H. Nguyen, S. Forgo, J. Schneider, J. Schrer, C. Krger, H. Matzke, R. O. Clment, J. Krause, and R. Rojas, Interactive robotic fish for the analysis of swarm behavior. in ICSI (1), ser. Lecture Notes in Computer Science, Y. Tan, Y. Shi, and H. Mo, Eds., vol. 7928. Springer, 2013, pp. 110.

[13] F. Bonnet, P. Rétornaz, J. I. Halloy, A. Gribovskiy, and F. Mondada, Development of a mobile robot to study the collective behavior of zebrafish, in IEEE International Conference on Biomedical Robotics and Biomechatronics, BioRob 2012, 2012.

[14] F. Bonnet, S. Binder, M. Elias de Oliveira, J. Halloy and F. Mondada. A Miniature Mobile Robot Developed to be Socially Integrated with Species of Small Fish. IEEE International Conference on Robotics and Biomimetics, Bali, Indonesia, 2014.

[15] P. R. Bandyopadhyay. Trends in biorobotic autonomous undersea vehicles. IEEE Journal of Oceanic Engineering, 2005, 30, 109139.

[16] Seok Heo, Tedy Wiguna, Hoon Cheol Park and Nam Seo Goo. Effect of an Artificial Caudal Fin on the Performance of a Biomimetic Fish Robot Propelled by Piezoelectric Actuators, Journal of Bionic Engineering 4 (2007) 151158

[17] Zhenlong Wang, Guanrong Hanga, Jian Li, Yangwei Wang and Kai Xiao. A micro-robot fish with embedded SMA wire actuated flexible biomimetic, Sensors and Actuators A 144 (2008) 354360. 